\title{
Guest Reviewers
}

This list reflects guest reviewers who completed a review for Cancer Biomarkers between September 18, 2014 and October 22 ${ }^{\text {nd }}, 2015$. We are appreciative of the time and effort these external reviewers devoted to providing expert feedback to our authors.

Fariba Abbasi

Dale Abrahamson

Marian Adamkov

Prasad Adusumilli

Irina Agoulnik

Farid Ahmed

Susanna Akiki

Edita Aksamitiene

Malcolm Alison

Deborah Altomare

Surya Amarachintha

Pouyan Amini Shakib

Dana Andersen

Karen Anderson

Ravindran Ankathil

Christina Annunziata

Katsuyuki Aozasa

Rami Aqeilan

Natalia Araujo

Yoshiaki Arimura

Samir Awadallah

Turkan Aytekin

Claudia Bănescu

Hideo Baba

Jaap Willem Back

Caleb Bailey

Nathan Baker

Margit Balázs

Sreeparna Banerjee

Balázs Bánky

Punit Bansal

Laura Barkley

Ana Battastini

Dalel Ben Néjima

Daniel Benharroch

Adam Berger

Gurå Bergkvist
Daniel Galera Bernabé

Dace Berzina

Ali Bettaieb

Mike Birrer

Meera Bista

Jan Blaakaer

Giovanni Blandino

Ivan Blasutig

Linda Bloom

Richard Blouin

Jesper Bonde

Edyta Borkowska

Lucia Borriello

Ugur Boylu

Cameron Bracken

Nathalie Bravenboer

Walburgis Brenner

M. Mitzi Brentani

Jan Brezovsky

Holger Bronger

Anna Brunet Vega

Barna Budai

Octavio Caba

Gabriella Cadoni

Stefano Cafarotti

Fabio Campodonico

Carlos Canto

Angelica Cappellari

Damiano Caputo

William Carson, III

Lina Carvalho

Raquel Catarino

Bryce Chackerian

Kishore Challagundla

Roger Chammas

Wen-Wei Chang

Pithi Chanvorachote

Lily Chen

Chun Yan Chen

Moonjae Cho

Naga Sirisha Chundru

Emilio Ciusani

Colin Clarke 
Christelle Clément-Duchêne

Tess Clendenen

William Coleman

Fabio Coppedè

Niall Corcoran

Daniel Costa

Gabriella Czifra

Nejat Dalay

Louise Dalgaard

Kaid Darwiche

Nurlan Dauletbaev

Jon Davison

Rosângela de Andrade

Anne Dee

Dilaver Demirel

Kristoffer Derwinger

Vanessa Deschoolmeester

Silvia Di Agostino

Nick Di Girolamo

Nyla Dil

Ying Ding

Marius Distler

Yavuz Dodurga

Peixin Dong

Vesna Dragutinovi? Alexandru Dregan

Laurent Dubuquoy

Frédéric Ducongé

Jozsef Dudás

Magdalena Dutsch-Wicherek

Gamal Ebid

Paolo Edomi

Aron Eklund

Maha Hamdi El Sissy

Hatem EL-mezayen

Fathia El-Sharkawi

Domagoj Eljuga

Ayse Engin

Monica Engstrøm

Francisco Enguita

Philipp Erben

Kati Erdmann

Irene Esposito

Ahmed Essaghir

Birthe Fahrenkrog

Yi-Mei Fan

David Faraoni

David Feliciano

Ziding Feng

Cristiano Ferlini

Francesca Ficara

Giulia Fontemaggi
Patrice Forget

Michael Froehner

Takaaki Fujii

Yutaka Fujiwara

Hiroshi Fukamachi

Andrea Fuso

Rajesh Gacche

Apar Ganti

Rocío García-Becerra

Pallavi Garg

Maria Gazouli

Niklas Gebauer

Elena Gershtein

Goutam Ghoshal

Benjamin Gibert

Yori Gidron

Antonio Giordano

Ioannis Gioulbasanis

Anna Git

Jianping Gong

William Grant

Dimitra Grapsa

Christophe Grosset

Wenyi Gu

Chunhua Guo

Yuni Elsa Hadisaputri

Sebastian Haen

Batoul Sadat Haerian

Roman Hajek

Chisato Hamashima

Xiuzhen Han

Daijiro Harada

Agnieszka Harazin-Lechowska

Robin Haring

Paul Hartel

Mandana Hasanzad

Mohammad Hashemi

M. Mathieu Hatt

Gerwin Heller

Ingegerd Hellstrom

Dov Hershkovitz

Lisa Hess

Dominique Heymann

Masanori Hisaoka

Ricardo Hitt

Maarten Hoek

Robert Hoffman

Stefan Holdenrieder

Kazufumi Honda

Chia-Ling Hsieh

Mei-Yu Hsu 
Jian-Kun $\mathrm{Hu}$

Xin Huang

Carolyn Hutter

Khadiga Ibrahim

Péter Igaz

Hisae Iinuma

Hisao Imai

Toshihiko Imamura

Manal Fouad Ismail

Jon Jacobs

Jens Jakob

Rahman Jamal

Maurice Jansen

Hae Myung Jeon

Li Jiao

Zhiming Jin

Duncan Johnstone

Ivana Joksic

Kimberly Jordan

Aurelija Juèaite

Csaba Juhász

Klaus Jung

Wajih Kaabachi

Christina Kalpadakis

Seok-Gu Kang

Gyeong Kang

Soundarapandian Kannan

Wynn Kao

Niki Karachaliou

Anastasia Kariagina

Sajjad Karim

Noriyuki Kasai

David Kashatus

Usha Kasid

Hideki Kawai

Mahmood Kayani

Julhash Kazi

Brett Keeling

Daniel Keizman

Abdelouahed Khalil

Alan Soo-Beng Khoo

Eiji Kikuch

Shojiro Kikuchi

Hyun-Seok Kim

In Joo Kim

Heui-Soo Kim

Jae-Sung Kim

Heon Kim

Yasuhide Kitagawa

Eugenie Kleinerman

Vera Kloten
Stian Knappskog

Ulker Kocak

Elise Kohn

Takashi Kohno

Emin Turkay Korgun

Serdal Korkmaz

Subramanian Krishnakumar

Balaji Krishnamachary

Sarawut Kumphune

Juozas Kupcinskas

Sven Kurbel

Anastasios Kyriazoglou

François Labrousse

Nathalie Lamarche-Vane

Paul Lampe

Yong Sun Lee

Jeeyun Lee

Ju-Seog Lee

Yi-Jang Lee

James Lee

Michael Leitzmann

Fabienne Lesueur

Euphemia Leung

Victor Levenson

Jinyan Li

Lin $\mathrm{Li}$

Cuiling Li

Hai Lian

In Kyoung Lim

Li Lin

Ren-Jang Lin

Fumin Lin

Kwang-Huei Lin

Alexander Link

Faina Linkov

Gui-Jian Liu

Liwei Liu

Gang Liu

Ko-Jiunn Liu

Yunhua Liu

Tao Liu

Xiuli Liu

Yuchen Liu

Bannakij Lojanapiwat

Anna Lokshin

Aurelio Lorico

Attila Lorincz

Manal Louis Louka

Maria Lozano

Antonio Lozano-Leon

Antonio Lucacchini 
Marta čuĹukaszewicz-Zając

Shao-gang Ma

Shaogang Ma

Antonio Macciò

Sabhiya Majid

Zahra Maleki

Anwar Mall

Michael Markey

Michele Markstein

Marie-Claire Maroun

Dana Marshall

John Martens

Eva Katharina Masel

José Mato

Andrea Mazzatenta

Damian Medici

Catharina Medrek

Parvin Mehdipour

Patrick Mehlen

Mario Menschikowski

Mohamed Meselhy Eltoukhy

Lisa Mestrinho

Junsei Mimura

Thomas Miner

Divya Mishra

Rama Mittal

Yasuhiko Mohri

Mathias Montenarh

David Moore

Massimo Moretti

Leslie Muldoon

Filip Mundt

Mogana Murtey

Pelin Mutlu

Manabu Muto

Leandro Müller

Urs Müller-Richter

Masaki Nagata

Siddavaram Nagini

Misa Nakamura

Gopeshwar Narayan

Silvio Naviglio

Lungile Ndlovu

Sarah Newbury

Joanna Niemiec

Takashi Nojiri

Brian Nolen

Yasuyuki Nomura

Katie O'Brien

Takahiro Ochiya

Shuji Ogino
Nobuo Ohta

Katsuhiro Okuda

Hiroshi Okumura

Mana Oloomi

Maxwell Omabe

Ola Omran

Mayumi Ono

Tarig Osman

Tomoko Oya-Ito

Fusun Ozmen

Narayanan Sathiya Pandi

Narasimha Reddy Parine

Dong Soo Park

Yongseok Park

Dinah Parums

Harvey Pass

Theodoros Pavlidis

Mary Ellen Pavone

Li Peipei

Peng Loh

Tiago Pereira

Songsak Petmitr

Nina Petroviç

Vladislav Petyuk

Raymond Pierce

Ruben Pio

Daniela Planska

Ruben Plentz

Ludmila Prokunina-Olsson

Milos Radojevic

Arvind Ramanathan

Cristiane da Fonte Ramos

Muhammad Usman Rashid

Madhup Rastogi

Robert Rebhun

Rishindra Reddy

Damon Reed

Henning Reis

Leo Reis

Sabrina Reis

Flávio Reis

Kenneth Remy

Tao Ren

Diana Reséndez-Pérez

Caroline Reuter

Alfredo Ribeiro-Silva

Carmela Ricciardelli

João Rodrigues

Fernando Rodríguez-Serrano

Achmad Chusnu Romdhoni

Carmen Romero 
Sandra Romero-Cordoba

Jason Ross

Charles Rosser

Simona Rossi

Giulio Rossi

Joshua Roth

Marie-Christine Rousselet

Carlos Rovira

Francesca Ruberti

Kathy Ruble

Nadia Rucci

Noha Mohamed Said

Takeshi Saito

Jun Sakakibara-Konishi

Takashi Saku

Andrea Salmaggi

Zahoor Samra

Joaquín Sánchez-Garcia

Monica Sankhwar

Raffaela Santoro

Leticia Santos

Sankar Nath Sanyal

Ana Bela Sarmento-Ribeiro

Manchanahalli Sathyanarayana Rao

Barbara Savoldo

Fatemeh Sayehmiri

Hans-Joachim Schmoll

Regine Schneider-Stock

Peter Schraml

Martin Schreiber

Heidi Schwarzenbach

Andreas Schüring

Durairaj Sekar

Annalucia Serafino

Alessandro Sgambato

Ivan Shabo

Alexandra S. Shadrina

Parantu Shah

Shohreh Shahabi

Kumaran Shanmugam

Rong Shao

Kiran Sharma

Rohini Sharma

Tomoyuki Shibata

Yohei Shimono

Bong Kyung Shin

Hirokazu Shoji

Michael Shurin

Rosalia Simmen

David Simpson

Sveinung Sorbye
Meindert Sosef

Melissa Southey

Liudmila Spirina

Alagarsamy Srinivasan

Kshitij Srivastava

Cathy Staedel

Athena Starlard-Davenport

Tomas Stopka

Stanley Stylli

Dhivya Sudhan

Hatuhiko Sugimura

Young Suh

Yongjoon Suh

Prasit Suwannalert

Kazuhiro Suzuki

Motofumi Suzuki

Hiroyuki Suzuki

Kris Szyfter

Michael Tainsky

Emanuela Taioli

Akinobu Takaki

Nagio Takigawa

Motohiro Tamiya

Ruo-Ying Tan

Yoichi Tanaka

Pierfrancesco Tassone

Kristina Thiel

Peter Thomas

Ling Tian

Marianne Tinguely

Katsuji Tokuhara

Marco Tomasetti

Takeshi Tomonaga

Maria Lina Tornesello

Valérie Trichet

Hans-Ingo Trompeter

Takahiro Tsuchikawa

George Tuszynski

Vibha Uppal

Narikazu Uzawa

Marc Philippe van der Schee

Frank Vari

Daniele Vergara

Renata Veselska

Julien Vincenten

Michelle Visser

Elena Voropaeva

Zeljko Vujaskovic

Marijana Vujkovic

Katarzyna Walczak

Nathan Wall 
Xiaoping Wan

Rongquan Wang

Wei Wang

Shukui Wang

Ying-Jie Wang

Kai Wang

Jianliu Wang

Dong Wang

Georg Weber

Daniel Weber

Thomas Weber

Stephanie Weinstein

Stefan Weiss

Piotr Wierzbicki

Nana Wilson

Jing Wu

Jun-Hua Wu

Hua-Sheng Xiao

Rui Xie

Edith Xing

Binghe $\mathrm{Xu}$

Seiichi Yamano

Hongwei Yang

Shao-Di Yang

Hiroyuki Yasuda
Eugenia Yazlovitskaya

Chen-Hsiang Yeang

Eugenia Yiannakopoulou

Jun Yin

Y. X. Yin

Gregory Yochum

Puangrat Yongvanit

Yoichiro Yoshida

Matthew Young

Yinghao $\mathrm{Yu}$

Kun-Hsing Yu

Helmut Zarbl

Paul Zarogoulidis

Anthony Zeitouni

Bai Zhang

Xin Zhang

Yuanzhen Zhang

Qunzhou Zhang

Xinna Zhang

Zhiyong Zhang

Lili Zhao

Wei Zhao

Hui Zheng

Ying Zhou

Xinchun Zhou 